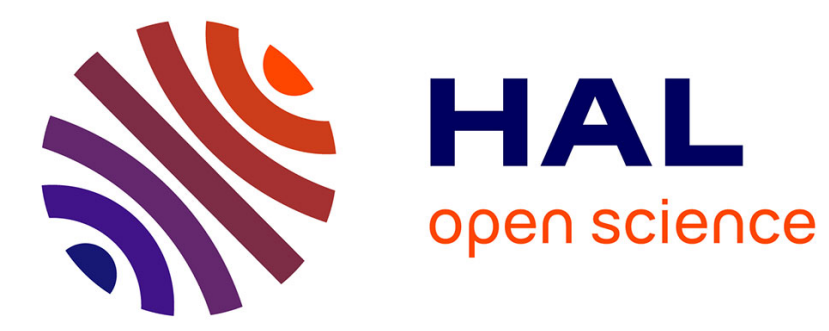

\title{
Classement syntaxique des prépositions simples du français
}

Céline Vaguer

\section{To cite this version:}

Céline Vaguer. Classement syntaxique des prépositions simples du français. Langue française, 2008, 157, pp.20-36. hal-00980412

\section{HAL Id: hal-00980412 \\ https://hal.science/hal-00980412}

Submitted on 17 Apr 2014

HAL is a multi-disciplinary open access archive for the deposit and dissemination of scientific research documents, whether they are published or not. The documents may come from teaching and research institutions in France or abroad, or from public or private research centers.
L'archive ouverte pluridisciplinaire HAL, est destinée au dépôt et à la diffusion de documents scientifiques de niveau recherche, publiés ou non, émanant des établissements d'enseignement et de recherche français ou étrangers, des laboratoires publics ou privés. 


\title{
Classement syntaxique des prépositions simples du français 1
}

\begin{abstract}
À notre connaissance, il n'existe pas d'études qui aient constitué l'inventaire des structures syntaxiques admises (ou exclues) pour chacune des prépositions du français ${ }^{2}$. Si, dans le cadre d'une linguistique descriptive, les travaux de Maurice Gross (1975) ont pu aller dans ce sens, le classement concernait les verbes et reposait sur des propriétés non étayées par des attestations pour chacune des entrées mentionnées. Ludo Melis, dans son recueil de 2003, a commencé un tel travail de recensement des propriétés syntaxiques caractérisant la préposition en général et chaque préposition en particulier mais sans que l'on aboutisse à un classement systématique. Il en va de même de Denis Le Pesant (2006) qui toutefois établit des corrélations entre les propriétés syntaxiques et distributionnelles d'une part, et les classes sémantiques d'autre part.

Notre propos est à la fois plus ambitieux et plus modeste que celui de ces prédécesseurs : plus ambitieux parce que nous visons à établir un panorama des propriétés syntaxiques que nous appliquerons à toutes les prépositions de manière à en établir le classement formel (à la manière de Maurice Gross pour les verbes), et que chacune des possibilités sera vérifiée sur un corpus attesté (FRANTEXT, GEOPO...). Plus modeste parce que nous ne parvenons pas, par là, à saisir le fin de l'identité de chaque préposition elle-même (car il faut pour cela procéder à l'étude des distributions dans leur détail).

De plus, il ne s'agit que d'un premier inventaire fondé sur deux corpus chacun dépouillé exhaustivement, certes, mais qui ne garantissent pas en eux-mêmes la représentativité des propriétés syntaxiques (complémentation (i.e. sous-catégorisation) et modification) observées - même de très gros corpus n'illustrant pas forcément le tout des possibilités permises par la langue.
\end{abstract}

1. Nos remerciements vont à Danielle Leeman et à Belinda Lavieu ainsi qu'aux deux relecteurs anonymes de la revue.

2. Or ce type d'information est nécessaire dans le domaine du traitement automatique de la langue, permettant d'affiner les analyseurs syntaxiques notamment dans bon nombre de programmes et logiciels. 
À partir de la liste des vingt-six prépositions simples proposée par Ludo Melis (op. cit. : 1053) et des études et supports existants (dictionnaires, grammaires, entre autres), nous présenterons dans un premier temps les données (corpus et outils linguistiques) sur lesquelles nous avons fait reposer notre étude. Puis, après avoir précisé comment s'est opérée la sélection des prépositions simples, nous dresserons un panorama des principales propriétés syntaxiques observées pour chacune de ces prépositions. Cette observation sera complétée par une étude sur corpus plus fine, i. e. centrée sur chacune des prépositions dans diverses sources sur une période considérée (afin d'être proche de l'exhaustivité par rapport à l'objet de recherche et la délimitation du corpus retenu). Dans des travaux ultérieurs, l'objectif est d'étendre le corpus afin d'aboutir à un classement syntaxique des prépositions simples du français prenant en compte l'ensemble des propriétés observées. L'idée sous-jacente est d'établir un rapport entre forme et sens : est-ce que des prépositions qui ont des comportements syntaxiques similaires peuvent également connaître des ressemblances sémantiques? Nous considérons comme Maurice Gross (1975 : 10) que « seule une accumulation de ce type de données permettra de développer des considérations pouvant conduire à la formulation de théories ».

\section{MÉTHODES DE RECUEIL ET PREMIÈRES OBSERVATIONS}

Afin de réunir les chances de rassembler un ensemble le plus représentatif possible des constructions syntaxiques que connaissent les prépositions simples $\mathrm{du}$ français, nous avons diversifié les corpus retenus puisque, comme le remarque Ludo Melis (2003: 18), «la valence ne se détermine ni au niveau de la classe, ni au niveau des lexèmes, mais en relation avec les emplois ». Nous sommes partie du Corpus GEOPO proposé par l'Institut Français des Relations Internationales (IFRI). Ce corpus, constitué d'environ 270000 mots, regroupe trente-deux textes longs qui sont des articles expositifs (informatifs et argumentatifs) proposant des réflexions relatives à notre monde d'aujourd'hui (la crise pétrolière, la guerre contre "l'axe du mal », le terrorisme, l'explosion chinoise, la paix au Moyen-Orient, etc.).

L'investigation a été complétée par des requêtes sur la base textuelle FRANTEXT, version non catégorisée, limitées à l'année 1995 pour l'ensemble des prépositions simples du français, afin d'avoir un panorama de la langue littéraire en synchronie contemporaine. Le présent corpus est ainsi constitué de sept ouvrages qui représentent 686652 mots, à quoi s'ajoute la consultation de chacune des entrées du Trésor de la langue française informatisé (TFLI) pour les prépositions simples du français retenues.

Pour mener à bien l'exploration de ces corpus, nous avons eu recours à Unitex ${ }^{4}$. A ainsi été créé, pour chacune des dites prépositions, un transducteur

3. à, après, avant, avec, chez, contre, dans, de, depuis, derrière, dès, devant, en, entre, envers, hors, jusque, par, parmi, pendant, pour, sans, selon, sous, sur, vers.

4. Unitex est un logiciel, open source, d'analyse de corpus linguistiques, qui intègre des dictionnaires électroniques et différents composants nécessaires à l'analyse morphologique et syntaxique des textes du corpus. 
à états finis, qui permet de façon automatique d'une part d'identifier les prépositions dans les corpus, d'autre part de les extraire et ainsi d'en proposer des concordances (ce qui nous permet d'avancer dans la modélisation des connaissances). Ont ainsi été extraites automatiquement dans les corpus GEOPO et FRANTEXT un certain nombre d'attestations (Tableau 1) pour chacune des prépositions simples retenues, correspondant à 100746 occurrences.

Il apparaît alors que l'ensemble des prépositions simples constitue plus de $13 \%$ du corpus GEOPO et plus de $9 \%$ de FRANTEXT. Il pourrait être également intéressant d'opérer des analyses de fréquence selon le genre (littéraire, journalistique...) du texte retenu. D'ores et déjà, la comparaison avec les fréquences enregistrées au titre du Français fondamental (Brunet 1981; Picoche 1993)

Tableau 1 : Occurrences des prépositions simples du français dans Geopo et Frantext

\begin{tabular}{|c|c|c|}
\hline \multirow[t]{2}{*}{ Prépositions simples } & \multicolumn{2}{|c|}{ Nombre d'occurrences } \\
\hline & GEOPO & FranteXt \\
\hline$A$ & 5421 & II 438 \\
\hline Après & 208 & 688 \\
\hline Avant & 155 & 458 \\
\hline Avec & 669 & 2411 \\
\hline Chez & 28 & 332 \\
\hline Contre & 390 & 362 \\
\hline Dans & 2280 & 5484 \\
\hline De & 14327 & 23205 \\
\hline Depuis & 333 & 445 \\
\hline Derrière & 13 & 260 \\
\hline Dès & III & 169 \\
\hline Devant & 65 & 360 \\
\hline$E n$ & 4277 & 6941 \\
\hline Entre & 588 & 483 \\
\hline Envers & II & 39 \\
\hline Hors & 39 & 51 \\
\hline Jusque & $|5|$ & 46 \\
\hline Par & 1958 & 2114 \\
\hline Parmi & 52 & 90 \\
\hline Pendant & 103 & 220 \\
\hline Pour & I 777 & 3853 \\
\hline Sans & 408 & 1523 \\
\hline Selon & 147 & 95 \\
\hline Sous & 226 & 640 \\
\hline Sur & 1689 & 3116 \\
\hline \multirow[t]{2}{*}{ Vers } & 100 & 396 \\
\hline & 35527 & 65219 \\
\hline
\end{tabular}


montre que celles obtenues sur les corpus FRANTEXT et GEOPO n'en sont pas si éloignées (comme l'illustre le classement des prépositions selon leur fréquence dans le Tableau 2). Seules différences majeures, les fréquences de chez et jusque: placées dans les quinze prépositions les plus fréquentes pour le Français fondamental, elles apparaissent comme des prépositions peu fréquentes dans les corpus consultés ( $18^{\mathrm{e}}$ rang pour chez, $25^{\mathrm{e}}$ pour jusque). De même, si la préposition envers se trouve illustrée dans nos corpus, sa fréquence reste marginale $(0.05 \%$ des emplois observés) - ce qui corrobore les données du Français fondamental, dans lesquelles elle ne figure pas. En effet, ce dernier retient les fréquences jusqu'au rang 907 (c'est-à-dire les mots de fréquence supérieure à 7 000).

Tableau 2 : Classement des prépositions simples du français par fréquences décroissantes

\begin{tabular}{|c|c|}
\hline \multicolumn{2}{|c|}{ Fréquences décroissantes } \\
\hline FRANÇAIS FONDAMENTAL & GEOPO/FrANTEXT \\
\hline$d e$ & $d e$ \\
\hline$\grave{a}$ & $\grave{a}$ \\
\hline en & en \\
\hline dans & dans \\
\hline pour & pour \\
\hline sur & sur \\
\hline par & avec \\
\hline avec & par \\
\hline sans & sans \\
\hline sous & après \\
\hline après & sous \\
\hline chez & entre \\
\hline jusque & avant \\
\hline entre & depuis \\
\hline vers & vers \\
\hline depuis & contre \\
\hline devant & devant \\
\hline contre & chez \\
\hline avant & derrière \\
\hline pendant & pendant \\
\hline dès & dès \\
\hline derrière & selon \\
\hline parmi & parmi \\
\hline selon & hors \\
\hline \multirow[t]{2}{*}{ hors } & jusque \\
\hline & envers \\
\hline
\end{tabular}


Insistons sur le fait que les résultats qui suivent ne sont exhaustifs que par rapport aux corpus retenus : nous ne prétendons donc pas avoir couvert l'ensemble des emplois possibles pour chacune des prépositions, mais seulement ceux qui sont attestés dans les trois sources ici analysées - nous pourrions dire à l'instar de Maurice Gross $(1975: 10)$ : «les données que nous présentons ne peuvent être considérées que comme une étape modeste dans cette direction, mais elles nous ont déjà permis de faire des observations d'un type nouveau [...] La systématicité de nos données nous a permis d'établir des corrélations entre formes qu'il n'était pas possible d'obtenir sans des études quasi exhaustives. La découverte d'autres phénomènes de ce type devrait donc avoir des conséquences importantes pour la linguistique théorique ».

\section{AVERTISSEMENT SUR LE CORPUS DES PRÉPOSITIONS}

\section{I. Sur la sélection opérée par Ludo Melis}

Ainsi que mentionné supra, nous retenons comme objet d'étude les vingt-six prépositions du français considérées par Ludo Melis (2003:105) comme les " plus typiques, c'est-à-dire les prépositions simples, qui apparaissent au locuteur comme inanalysables » (Ibid.). La sélection a écarté, relativement à la liste que donnent généralement les ouvrages de référence, plus particulièrement:

- les prépositions issues d'anciens participes: concernant, durant, moyennant, nonobstant, suivant, touchant ; attendu, excepté, passé, supposé, vu, hormis ;

- les adjectifs qui connaissent un emploi de préposition : sauf ${ }^{5}$, plein...;

- certains adverbes comme près...;

- les prépositions en voie de disparition: deçà, delà, dedans, dessous, dessus, devers, dont certaines peuvent fonctionner soit comme adverbe, soit comme nom ;

- les prépositions proches de locutions prépositives comme quant à...

Si l'ensemble de ces items ont été écartés par Ludo Melis de sa sélection des prépositions (et dans le présent article pour des raisons éditoriales), il va sans dire qu'elles mériteraient à leur tour une étude approfondie comme celle que nous proposons pour les prépositions dites « simples ».

\subsection{Une préposition peut en cacher une autre}

Certaines prépositions simples ont un double fonctionnement.

5. Sauf peut être identifié soit comme une préposition, soit comme un adjectif. Du point de vue diachronique, l'emploi prépositionnel est dérivé de l'emploi adjectival - c'est pourquoi nous parlons ici d'«adjectifs qui connaissent un emploi de préposition». 


\subsection{Le cas de jusque : préposition ou spécificateur ? ${ }^{6}$}

La forme de surface (jusque + Prép) reçoit deux analyses, jusque pouvant être identifié soit comme préposition, soit comme spécificateur.

- La préposition jusque, en tant que spécificateur, vient modifier les prépositions chez, dans, derrière, sous et sur.

[1] a. Pour la signature de l'acte, le notaire vint jusque chez elle. [Frantext]

b. Jusque dans ses conséquences sociales, le conflit est un succès. [Geopo]

c. On planquait le sel jusque derrière les autels, l'or dans les berceaux. [Frantext]

d. Du reste, le livre traînait sur toutes les toilettes, tous les chevets, et jusque sous les oreillers. [Frantext]

e. Le commis portait, jusque sur le visage, des plaies multiples, mais aucune ne paraissait profonde. [Frantext]

En tant qu'ajout, jusque est supprimable et apporte une information d'ordre aspectuel; ainsi la différence entre [2a] et [2b] :

[2] a. Le notaire vint chez elle.

b. Le notaire vint jusque chez elle.

est que dans [2b] jusque marque le parcours, l'étendue (on voit le notaire en train de venir) - ce qui rejoint l'analyse proposée par Danielle Leeman (2005) de jusque. Cette idée $\mathrm{d}^{\prime}$ " étendue» en tant qu'elle est non bornée favoriserait l'implication d'une " exagération " (Dictionnaire de Trévoux et Leeman 2005) -les énoncés [1b-e] supposent effectivement que ce qui se produit dépasse ce à quoi on peut s'attendre, ce qui est normalement envisageable.

- En tant que préposition, jusque + Prép forme un tout indissociable (jusque ne peut être supprimé $-3 b$ ). Dans cette locution, ce sont à et en qui apparaissent le plus souvent:

[3] a. Au plan des capacités civiles, la situation était plus satisfaisante dans la mesure où les Etats avaient confirmé leurs engagements de fournir jusqu'à 5000 policiers pour la mise en oeuvre du plan d'action de police adopté à Göteborg en juin 2001. [Geopo]

b. *.. les États avaient confirmé leurs engagements de fournir à 5000 policiers pour la mise en oeuvre du plan d'action de police adopté à Göteborg en juin 2001.

(fournir est transitif direct : ici c'est jusqu'à qui fonctionne comme spécificateur du syntagme nominal, comme le feraient presque ou environ). Dans cet emploi, la préposition composée jusqu'à « remplit les fonctions de marqueur de dynamicité, d'intensifieur (éventuellement argumental), d'emphatiseur, et de marqueur aspectuel » (Fortis 2006).

6. Pour une présentation détaillée des différents cas de figure, cf. Leeman 2005 et Fortis 2006. 


\subsubsection{Hors et hors de}

De même, la forme hors connaît plusieurs emplois. On a ainsi deux formes hors et hors de - évidemment liées morphologiquement - qui ne sont pas de simples variantes, comme le montrent les impossibilités de commutation :

[4] a. Elle se leva, hors d'elle. [Frantext]] *Elle se leva, hors elle.

b. Il respecte toutes les femmes, hors elle./ 'Il respecte toutes les femmes, hors d'elle.

Dans le premier cas, hors de marque une localisation extérieure à ce que désigne son complément (une maison hors de Paris, c'est une maison qui se trouve à l'extérieur de Paris), tandis que dans le second cas, hors a valeur exceptive (équivalent : sauf) - c'est-à-dire la même valeur que précédemment mais transformée sur le plan notionnel - et le SP lui-même valeur énonciative (limitant la portée du reste du propos). Cependant l'opposition paraît neutralisée dans certains contextes (où hors de a valeur exceptive) :

[5] a. Je puis tout supporter, hors de pleurer loin de toi. [TLFi]

b. Je puis tout supporter, hors pleurer loin de toi.

Nous admettons provisoirement, pour cette étude, l'existence d'une préposition hors (de) car l'analyse du corpus s'est faite sur la forme hors et non sur l'existence de deux formes distinctes hors et hors de (pour une telle analyse, cf. Vaguer 2007).

\subsubsection{Les prépositions « discontinues »}

Certaines des prépositions identifiées comme des "prépositions simples » (à, avec, de, depuis, en, entre, pour, sans...) entrent dans la formation d'une unité actualisée de manière "discontinue » : de... à..., de... en..., entre... et..., parmi d'autres.

[6] a. En cela, il différait du National Space Council, qui a existé de 1958 à 1973, puis de 1988 à 1993. [Geopo]/ Il va de Nantes à Blois par Angers et Tours. [Melis, $2003: 69]$

b. Elle errait de château en château. [TLFi]

c. Du 11 septembre jusqu'à aujourd'hui, les événements ont partiellement redirigé les esprits vers l'extérieur et suscité une aubaine budgétaire. [Geopo]

d. Le globe a tourné, montrant tout le continent d'Asie, depuis l'Inde jusqu'à la Chine. [TLFi]

e. Entre Emile êt Alexis, il n'y avait d'opposés que le tempérament et le projet. [Frantext]

Puis il dut choisir entre la femme qu'il aimait et la boxe. [Frantext]

Ludo Melis les identifie comme des prépositions employées « en interposition ou en corrélation » $(2003: 35)$ qui jouent le rôle de déterminant de quantité approximative : 
[7] a. Les organisateurs attendent de 500 à 600 personnes.

b. On attendait entre 500 et 600 personnes.

mais, comme on peut l'observer sous [6], leur sémantique dépend de la distribution mise en place ; elle ne se limite donc pas à l'approximation.

Parmi ces prépositions discontinues, des associations non encore signalées comme telles pourraient prendre place: c'est le cas de avec... pour... [8] et sans... pour autant [9]. En effet, si pour $N$ ne peut être analysé comme complément de avec ou de sans - comme le montrent la séparation possible (par une pause ou une virgule [a]) et le déplacement [b] - on a quand même affaire à quelque chose qui ressemble (sans être aussi figé qu'elles) aux prépositions discontinues (de type de... en...), ou plutôt à ce que Claire Blanche-Benveniste range sous les «noyaux complexes» $(1990: 118)$ ou ce que la grammaire de constructions appelle, justement, des «constructions » - c'est-à-dire une sorte de cadre syntaxique avec un lexique partiellement fixe, le reste étant susceptible de varier librement, comme dans «plus + phrase, plus + phrase »: Plus je te vois, plus je t'aime/Plus il dort, plus il se sent fatigué, etc.

[8] Il fallait soit mettre en place un marché libre de la banane au sein de l'Union (avec pour effet l'éviction des producteurs européens locaux et de beaucoup de producteurs antillais). [Geopo]

a. Il fallait soit mettre en place un marché libre de la banane au sein de l'Union (avec, pour effet, l'éviction des producteurs européens locaux et de beaucoup de producteurs antillais).

b. ... avec l'éviction des producteurs européens pour effet...

[9] Robert Kagan rompt avec cette approche timorée et formaliste, sans pour autant tomber dans les excès ni dans l'analyse ni dans le diagnostic. [Geopo]

a. Robert Kagan rompt avec cette approche timorée et formaliste, sans, pour autant, tomber dans les excès ni dans l'analyse ni dans le diagnostic.

b. ... sans tomber dans les excès pour autant, ...

Notre analyse ne les prend pas en considération en tant que telles. Autrement dit, nous n'avons pas proposé d'un côté une étude de la préposition de, de l'autre une étude des emplois de de... en... puisque cela s'opposait à notre raisonnement de départ qui consistait à partir d'une forme (soit une préposition simple).

\subsection{Des emplois d'un autre type}

On admet qu'une préposition peut avoir un complément nul (emploi rangé traditionnellement dans celui des adverbes). Les emplois présentés sous [10] n'illustrent pas le cas d'un complément nul mais celui d'une coordination (devant le même complément): [interagissent avec et influent sur] les échelons supérieurs... (10a), pour ou contre (10b), [se greffer sur ou avoir lieu à] un moment où... (10c). 
[10] a. L'Union est un parfait exemple de système de gouvernance à plusieurs strates dans lequel les institutions politiques d'un niveau interagissent avec et influent sur les échelons supérieurs et inférieurs. [Geopo]

b. Chacun doit choisir son camp : pour ou contre la terreur. [Geopo]

c. L'éventualité d'un regain de tension violente au Liban acquerrait plus de consistance encore - mais aussi plus de complexité - si elle venait à se greffer sur, ou à avoir lieu à un moment où la situation interne syrienne risque de se compliquer en raison de la transition délicate que ne manquera pas d'ouvrir la succession du président Assad. [Geopo]

Conclusion. Dans le cadre de notre étude, nous avons retenu tous les emplois de jusque (tant comme spécificateur que comme préposition composée), de hors (comme préposition et locution prépositive) et de l'ensemble des prépositions simples pouvant aussi par ailleurs apparaître comme des prépositions discontinues. Les fréquences proposées dans le Tableau 1 rassemblent donc sous une même entrée ces doubles fonctionnements. Toutefois, nous pouvons préciser que parmi les emplois de jusque (197 occurrences), $14 \%$ correspondent à la fonction de spécificateur et $86 \%$ au statut de préposition composée ; $62 \%$ des emplois de hors sont de type hors de et $38 \%$ de type hors (ce qui tend à prouver que l'emploi de hors préposition s'amenuise au profit de hors de locution prépositive); la plupart des entrées de prépositions pouvant être analysées tant comme des prépositions simples que comme des prépositions discontinues n'illustrent que la première possibilité.

\section{PROPRIÉTÉS SYNTAXIQUES ET PROBLÈMES DE CLASSEMENT}

Les observations sur corpus ont permis d'une part de caractériser le rapport qu'entretient la préposition avec son complément, d'autre part d'identifier quels peuvent être ces compléments pour les vingt-six prépositions simples du français. Ainsi la préposition peut avoir pour complément un syntagme nominal, un syntagme prépositionnel, un infinitif, une sous-phrase introduite par que/ce que, un complément nul, entre autres.

Ces propriétés combinatoires permettent de rassembler sur le plan syntaxique des prépositions qui n'auraient pas été forcément regroupées (du moins intuitivement) sur le plan sémantique. Il convient donc de s'interroger sur ce regroupement syntaxique : pourquoi certaines prépositions si différentes sur le plan sémantique ont-elles les mêmes propriétés syntaxiques? Deux hypothèses de réponse :

- ou bien il n'y a pas de lien entre forme et sens, mais deux systèmes qui coexistent: d'un côté celui des propriétés syntaxiques, de l'autre celui de l'identité sémantique (cette hypothèse invalide la plupart des théories linguistiques, à commencer par le structuralisme tel qu'initié par Ferdinand de Saussure) ;

- ou bien il y a bien une relation consubstantielle entre forme et sens, mais l'identité sémantique que dessinent les propriétés formelles n'est pas 
directement accessible par l'intuition spontanée - c'est ce que montre le travail de Jean Dubois et Françoise Dubois-Charlier (1997) sur les verbes ${ }^{7}$. Cette hypothèse est, au contraire de la précédente, en cohérence complète avec les postulats saussuriens: la grille sémantique (propre à chaque langue) étant arbitraire (par rapport à notre (appréhension du) monde), les valeurs secrétées par le système ne correspondent pas directement à l'intuition référentielle et ne peuvent être construites par le linguiste qu'à partir des indices fournis par les formes observables et de leur interprétation (hypothétique).

Si l'on adopte cette seconde option, l'un des guides (heuristiques) pour une telle interprétation est de ne pas mettre toutes les propriétés formelles sur le même plan (principe adopté par Maurice Gross (op. cit.), afin de ne pas aboutir à un "classement» où chaque "classe " ne dépasserait pas la paire de mots). La question qui se pose alors est de savoir sur quel critère décider que telle propriété est (vraisemblablement) plus pertinente que telle autre pour situer la préposition dans le système.

Le tableau général non classé (sinon selon 1'ordre alphabétique, Tableau $3^{8}$ ) montre qu'il y a des propriétés plus ou moins définitoires : la sous-catégorisation d'un SN par la préposition est un critère général, qui permet de distinguer cette catégorie de toutes les autres hormis le verbe, susceptible également d'avoir un complément direct (cette propriété est néanmoins beaucoup plus caractéristique de la préposition, puisque certains verbes n'admettent qu'un complément indirect et que d'autres sont intransitifs alors que toutes les prépositions sont/+SN/). À partir de là, et selon le même raisonnement, on peut admettre que les propriétés les plus pertinentes sont celles qui sont les plus représentées dans ce tableau (pour le trait $/+\varnothing /$, on arrive aux chiffres ' 11 'sans tenir compte des formes dedans, dessous, dessus... et ' 15 'autrement), soit (par ordre décroissant) :

\begin{tabular}{|l|l|l|}
\hline Prép & + SN & 26 \\
\hline & $+P$ & 15 \\
\hline & $+\varnothing$ & II (15) \\
\hline & + SP & 9 \\
\hline & + SV $_{\text {inf }}$ & 9 \\
\hline
\end{tabular}

7. Cf. le numéro de Langue française 153, coordonné par J. François, D. Le Pesant et D. Leeman (2007), consacré à ce travail.

8. Nous mentionnions en introduction que les corpus consultés ne garantissaient pas en euxmêmes la représentativité des propriétés syntaxiques. Ainsi, le signe « - " qui apparaît pour chacune des propriétés syntaxiques non validées par une préposition ne l'est donc qu'à l'égard des corpus analysés. Certaines propriétés syntaxiques pourraient donc se trouver validées dans d'autres corpus : la préposition sous, par exemple, semble admettre un complément nominal en ce que comme le mentionne Danielle Leeman (2008): Il se réfugie sous ce que la loi l'oblige au devoir de réserve. 
Tableau 3 : Propriétés syntaxiques des prépositions simples du français

\begin{tabular}{|c|c|c|c|c|c|c|}
\hline & \multirow[t]{2}{*}{$+S N$} & \multirow[t]{2}{*}{$+S P$} & \multicolumn{2}{|c|}{$+P$} & \multirow{2}{*}{$\begin{array}{c}+ \text { SV } \\
\text { infinitif }\end{array}$} & \multirow{2}{*}{$\begin{array}{c}\text { + Complément } \\
\text { nul }\end{array}$} \\
\hline & & & que & ce que & & \\
\hline A & + & - & - & + & + & - \\
\hline Après & + & - & + & - & + & + \\
\hline Avant & + & + & + & - & + & + \\
\hline Avec & + & - & - & - & - & + \\
\hline Chez & + & - & - & - & - & - \\
\hline Contre & + & - & - & - & - & + \\
\hline Dans & + & - & - & + & - & - \\
\hline De & + & + & - & + & + & - \\
\hline Depuis & + & - & + & - & - & + \\
\hline Derrière & + & + & - & - & - & + \\
\hline Dès & + & - & + & - & - & - \\
\hline Devant & + & + & - & - & - & + \\
\hline En & + & + & - & + & - & - \\
\hline Entre & + & - & - & - & - & - \\
\hline Envers & + & - & - & - & - & - \\
\hline Hors (de) & + & + & + & - & + & - \\
\hline Jusqu'à & + & - & - & + & + & - \\
\hline Par & + & + & - & - & + & - \\
\hline Parmi & + & - & - & - & - & - \\
\hline Pendant & + & - & + & - & - & - \\
\hline Pour & + & + & + & - & + & + \\
\hline Sans & + & - & + & - & + & + \\
\hline Selon & + & - & + & - & - & + \\
\hline Sous & + & - & - & - & - & - \\
\hline Sur & + & - & - & + & - & - \\
\hline Vers & + & + & - & - & - & + \\
\hline
\end{tabular}

On dénombre une dizaine de combinaisons possibles (a priori les plus « productives ») qui permettent de regrouper les prépositions simples du français selon les types de complément qu'elles sous-catégorisent : les prépositions sont comme le verbe ou l'adjectif, elles sous-catégorisent leurs arguments (Harris 1976). L'analyse des corpus et des caractéristiques syntaxiques de chaque préposition aboutit aux classes suivantes de prépositions selon le type de constructions syntaxiques qui les caractérisent: 
1. Prépositions qui ont en commun/+SN/seulement: chez, entre, envers, parmi, sous

2. Prépositions qui ont en commun $/+\mathrm{SN} / \mathrm{et} /+\mathrm{P} /:$ dans, dès, pendant, sur

3. Prépositions/+SN/et/+Complément nul/ : avec, contre

4. Préposition/+SN/et/+SP/et/+P/: en

5. Préposition $/+\mathrm{SN} / \mathrm{et} /+\mathrm{SP} / \mathrm{et} / \mathrm{SV}_{\text {inf }} /:$ par

6. Prépositions/+SN/et/+SP/et/+Complément nul/ : derrière, devant, vers

7. Prépositions/+SN/et/+P/et/ $+\mathrm{SV}_{\text {inf }} /: \grave{a}$, jusqu'à

8. Prépositions $/+\mathrm{SN} /$ et $/+\mathrm{P} /$ et $/+$ Complément nul/ : depuis, selon

9. Prépositions/+SN/et/+SP/et/+P/et/ $+\mathrm{SV}_{\text {inf }} /:$ de, hors (de)

10. Prépositions $/+\mathrm{SN} / \mathrm{et} /+\mathrm{P} / \mathrm{et} /+\mathrm{SV}$ inf $/$ et $/+$ Complément nul/ : après, sans

11. Prépositions/+SN/et/+SP/et/+P/et/+SV inf $/$ et/+Complément nul/ : avant, pour

Certaines prépositions limitent leur combinatoire au syntagme nominal (comme chez, entre, envers, parmi, sous - soit $19 \%$ des prépositions étudiées), d'autres à deux types de complément (avec, contre, dans, dès, pendant, sur - soit $23 \%$ des prépositions étudiées) ou trois types de compléments (à, depuis, derrière, devant, en, jusqu'à, par, selon, vers - soit $35 \%$ des prépositions étudiées), d'autres enfin admettent une large gamme de compléments (comme après, avant, de, hors (de), pour, sans - soit $23 \%$ des prépositions étudiées). Ces propriétés combinatoires permettent de rassembler sur le plan syntaxique des prépositions qui n'auraient pas été spontanément regroupées sur le plan sémantique.

Il y en a notamment que l'on présente habituellement « en couple », comme sur/sous, avec/sans, après/avant, et qui ne relèvent pas de la même classe syntaxique; dans la mesure où ces «paires » semblent reposer sur une appréhension intuitive fondée sur certains exemples (jugés) prototypiques à partir d'une intuition référentielle (par exemple spatiale), il est confirmé que cette présentation, pour traditionnelle qu'elle soit, ne repose pas sur l'observation des comportements linguistiques (c'est-à-dire des formes). Cela rejoint les propos de Peter Blumenthal dans ce même numéro.

\section{I. Comparaison des emplois de/P/et/SV inf $_{\text {I }}$}

Toute préposition sous-catégorisant un complément de type $/ \mathrm{P} / \mathrm{n}^{\prime}$ en admet pas forcément un de type $/ \mathrm{SV}_{\text {inf }} /$; c'est le cas notamment de dans, depuis, dès, en, pendant, selon, sur. Mais une préposition susceptible de se construire avec un complément $/ \mathrm{SV}_{\text {inf }} /$ admet le plus généralement un complément $/ \mathrm{P} /$ (seul par fait exception). Et il y a des prépositions qui acceptent (à, après, avant, de, hors, jusqu'à, pour, sans) ou excluent les deux (avec, chez, contre, derrière, entre, envers, devant, parmi, sous, vers).

Que conclure de ce constat? Chez peut paraître purement «spatial» (en un sens "concret»: chez Paul ou "abstrait»: Chez Montaigne au sens "dans l'œuvre/la philosophie de Montaigne ») et exclut aussi bien/P/et/SV $\mathrm{inf}_{1} /$; mais c'est aussi le cas de contre, or contre a des emplois dits «notionnels » (l'opposition 
ou l'échange). On ne peut donc pas strictement relier au sens «spatial » l'exclusion de ces deux types de complémentation. De même depuis, dès, pendant sont seulement temporels et ont pour propriétés/+P/et $/-\mathrm{SV}_{\text {inf }} /$, tout comme dans, en, selon ou sur qui ne sont pourtant pas seulement temporels.

Il ressort de ces observations que les dénominations référentielles traditionnelles ne paraissent pas appropriées pour étiqueter sémantiquement les classes ou corrélations syntaxiques.

\subsection{Les prépositions qui supposent 'que $P$ ' vs 'ce que $P$ '}

Généralement, on associe la «variante » Prép + ce que aux prépositions dites «vides » (à, de, en), par opposition aux prépositions sémantiquement " pleines » qui admettraient le seul que (avant, après, pour...). Mais on observe ici un dans ce que (*dans que étant impossible) et Hava Bat-Zeev Shyldkrot dans le présent numéro relève également sur ce que - alors que ni dans ni sur ne sont considérées comme «vides ». Si corrélation il y a, elle n'est donc pas aussi simple que ce que véhicule la doxa.

[11] Toute la difficulté tient dans ce que les sociétés humaines ne prennent les catastrophes au sérieux que lorsqu'elles se produisent. [Geopo]

Il insiste beaucoup sur ce que ces deux imprudentes n'ont pas emmené de chauffeur. [Mauriac]

Au-delà de cette opposition (entre prépositions «vides» ou "pleines»), $l^{\prime}$ analyse du cotexte ${ }^{9}$ révèle que les constructions Prép + ce que sont étroitement liées à un verbe recteur et sont à identifier comme des " constructions complétives indirectes » (Muller, $2004: 440$ ). Ce n'est donc pas le type de préposition («vide», «pleine »...) qui justifie son emploi avec ce que ou que mais la sélection ou non par le verbe de la préposition. Ainsi la préposition entre en construction avec que (Prép + que) lorsqu'elle garde sa valeur prédicative (i.e. elle n'est pas sélectionnée par un verbe).

\subsection{Les prépositions qui admettent un SV comme complément}

Certaines prépositions ont pour propriété commune d'admettre un complément de type verbal: soit de la forme d'un participe présent exclusivement introduit par en [12], soit d'un infinitif (à, après, avant, de, hors (de), jusqu'à, par, pour, sans) [13-16].

[12] Certes, à Durban, les États-Unis ont fait une concession à l'air du temps en acceptant de s'« excuser» pour l'esclavage. [Geopo]

La propriété sépare clairement en et dans, traditionnellement étudiées l'une par rapport à l'autre (ce que contestent, pour d'autres raisons, Franckel \& Lebaud 1991).

9. Nous remercions un de nos relecteurs d'avoir attiré notre attention sur ce point. 
Pour l'infinitif, il convient de distinguer :

- les prépositions qui admettent l'infinitif (direct) - à, après, de, jusqu'à, par, pour, sans... - illustrées sous [13] :

[13] Les raisons exactes qui ont poussé Slobodan Milosevic à capituler font encore aujourd'hui l'objet de débats. [Geopo]

Ils arrivaient à l'église après avoir plumé les haies. [Frantext]

En étendant l'imposition, il serait sans doute aussi possible de baisser le taux de cet impôt. [Geopo]

En effet, la recherche de la modération ne va pas jusqu'à cultiver le consensus avec le parti adverse. [Geopo]

La procédure finit par devenir affaire de culture. [Geopo]

Il attend le moment opportun pour abattre une carte qu'il estime gagnante. [Geopo]

Le président avait la possibilité de prendre certaines initiatives dans l'urgence sans demander l'avis du Congrès. [Geopo]

- les prépositions qui n'admettent l'infinitif que de manière indirecte : *avant aborder/avant d'aborder. Prendre en compte cette propriété oblige à séparer avant de pour dans la classe 11 supra.

[14] Il convient donc de commencer par un état des lieux avant d'aborder successivement les aspects opérationnel et politique du problème. [Geopo]

- les prépositions qui admettent les deux (construction directe ou indirecte) : hors relire/hors de pleurer. Cette variante devrait conduire à distinguer hors des autres prépositions de la classe 9.

[15] En fait, il n'y a qu'une seule chose qu'elle sache faire, hors relire son pedigree: résister à la tentation d'avaler la mouche que lui a lancée le vieux gentleman [...]. [Frantext]

Je puis tout supporter, hors de pleurer loin de toi. [TLFi]

- la préposition avant s'oppose encore aux autres par la possible alternance avant de mettre, avant que de mettre... (On n'aurait pas * pour que de mettre.)

[16] Oui, docteur, ma mère est morte avant que de me mettre au monde. [Frantext]

\subsection{Les prépositions qui supposent une variation morphologique}

Certaines prépositions connaissent deux formes selon que le complément est réalisé (dans, hors, sous, sur) ou non (dedans, dehors, dessous, dessus). Si l'on neutralise cette variation morphologique, la possibilité d'admettre une complémentation nulle se révèle une propriété pertinente pour caractériser les prépositions (puisqu'elle se trouve illustrée pour $58 \%$ des prépositions simples). Le fait que les «formes longues » puissent apparaître avec un complément rend cette neutralisation relativement acceptable dans le cadre du classement syntaxique : Un cri étouffé leur parvient de dessous le canapé. 


\subsection{Les prépositions qui connaissent l'emploi nominal}

Parmi les prépositions simples ici analysées, certaines connaissent l'emploi nominal. C'est le cas notamment de: après, avant, derrière. Cette propriété sépare encore avant et après des autres prépositions rangées dans les classes 10 et 11, mais confirme la définition de la classe 6 (derrière et devant).

[17] L'on distingue et distinguera un «avant » et un "après ». [Geopo] Avec Lole, il y avait un après au plaisir. [Frantext]

Depuis, je vivais dans cet après. [Frantext]

$C^{\prime} e s t$ dans l'après de l'amour que, chez moi, tout se déglinguait. [Frantext]

Le derrière de la maison./ Le devant d'un corsage. [Grand Larousse de la langue française]

\subsection{Les autres compléments de la préposition}

Outre ces possibilités, une préposition peut aussi être susceptible d'avoir pour complément :

- un adverbe (à + jamais/aujourd'hui; avant + longtemps ; (quelqu'un) de + bien, de + demain; depuis + toujours/hier/lors/longtemps/peu; dès + aujourd'hui/demain/lors/à présent/maintenant; en + bref; jusqu'à + récemment/aujourd'hui; jusqu' + alors/ici ; jusque + là/tard; par + delà/làlailleurs ; pendant + longtemps ; pour + aujourd'huil demain/plus tard/longtemps; sans + plus...);

- un adjectif (peindre en rouge, dessiner en grand; encore un problème de résolu (Anscombre 2005) ; il passe pour gentil, laissé pour mort, pour intéressant qu'il soit ; c'était dur de chez dur...)

et même d'avoir deux compléments : l'un nominal, l'autre adjectival (après sa fortune faite, avec ma fille malade, dès la nouvelle connue).

La prise en compte de ces complémentations aboutit à modifier les rassemblements (1) à (11) précédents : dès ne peut plus, si l'on ajoute le critère du double complément, être rangée dans la classe 2 et sera séparée de surcroît de pendant (autre préposition seulement « temporelle »).

\section{CONCLUSION}

Il apparaît de cet inventaire systématique, dans un corpus d'environ 957000 mots, des 100746 occurrences des vingt-six prépositions simples retenues, que ces dernières représentent près de $11 \%$ des mots utilisés dans les discours (corpus GEOPO et FRANTEXT ici retenus), ce qui est loin d'être marginal compte tenu de la minuscule liste qu'elles constituent au regard des milliers de noms, adjectifs ou verbes que compte le français.

Les prépositions ont ici été classées selon les propriétés syntaxiques qu'elles manifestent dans le corpus en question, du point de vue de leur régime, c'està-dire du type de constituant qu'elles sous-catégorisent - étant entendu que cette sous-catégorisation ne leur est pas uniquement imputable, dépendant 
aussi de la tête dont elles introduisent le complément (cf. la présentation de Danielle Leeman et aussi la contribution de Gerda Haßler et François Muller à ce même numéro). Il s'avère que le classement ainsi obtenu ne valide pas les regroupements sémantiques habituellement opérés, sauf pour derrière/devant (classe 6), traditionnellement rapprochés en tant qu'ils forment un couple d'antonymes d'ordre spatial (à l'aune de l'intuition référentielle éventuellement confirmée par le test de la question où ?).

On pourrait sur cette base considérer devant/derrière comme prototypiques des prépositions locatives, cependant la combinaison de leurs trois propriétés syntaxiques définitoires ne se retrouve pas dans les emplois des autres prépositions dites "spatiales »-ainsi, chez ou parmi ne sous-catégorisent ni/SP/ni /Complément nul/, à l'inverse de pour, qui n'est pas spécialement locative.

Le problème est alors posé du statut relatif de la forme et du sens, dont le type de résolution conditionne la suite du travail de recherche : ou bien l'observation n'est pas étonnante si l'on admet que la forme et le sens constituent chacun un système autonome ; il n'y a donc pas à être surpris que rien dans la syntaxe ne vienne confirmer l'intuition sémantique. Ou bien au contraire on considère que c'est la langue elle-même qui constitue un système autonome, dont le fonctionnement est assuré par les interrelations entre formes et sens (alors à considérer non arbitraires), indépendamment de l'organisation du monde tel qu'il existe objectivement ou tel qu'on se le représente conceptuellement. Il s'agit alors d'étudier un autre aspect de la forme : celui des possibilités et impossibilités distributionnelles, afin de voir ce que permettent de conclure les paradigmes lexicaux sélectionnés dans chaque cas de figure.

\section{Références bibliographiques}

AnsCOMBRE, J.-C. (2005) «Temps, aspect et agentivité dans le domaine des adjectifs psychologiques », LIDIL, 32 (145-166).

BAT-ZEEV SHYLDKROT, H. (2008) « Complétives introduites par Prép que $P$ vs complétives introduites par Prép à ce que $P »$, Langue française, ce numéro.

BLANCHE-BENVENISTE, C. (1990) Le français parlé. Etudes grammaticales, Paris, CNRS éditions.

BLUMENTHAL, P. (2008) «Combinatoire des prépositions : approche quantitative », Langue française, ce numéro.

BRUNET, E. (198I) Le vocabulaire français de 1789 à nos jours d'après les données du 'Trésor de la langue française', Genève-Paris, Slatkine-Champion.

DUBOIS, J. \& DUBOIS-CHARLIER, F. (1997) Les verbes français, Paris, Larousse-Bordas.

FORTIS, J.-M. (2006) «Les fonctions de jusqu'à », Modèles linguistiques, Tome XXVII-2, vol. 54 (I37- I54).

FRANCKEL, J.-J. \& LEBAUD, D. (199I) «Diversité des valeurs et invariance du fonctionnement de en préposition et pré-verbe », Langue française, 91 (56-79).

FrançoIs, J., LE PESANT, D. \& LEEMAN, D. (éds) (2007) Langue française n 153 : Le classement syntactico-sémantique des verbes français, Paris, Larousse.

Gross, M. (1975) Méthodes en syntaxe, Paris, Hermann.

HARRIS, Z.-S. (1976) Notes du cours de syntaxe, Paris, Le Seuil.

HaßleR, G. \& MUlleR, F. (2008) «Théorie et description des prépositions dans la linguistique romane en Allemagne », Langue française, ce numéro. 
LE PESANT, D. (2006) «Esquisse d'une classification syntaxique des prépositions simples du français », Modèles linguistiques 53, Tome XXVII-I (5I-74).

LeEMAN, D. (2005) «La préposition jusque », in P. Dendale (éd) Le mouvement dans la langue et la métalangue, Metz, Université de Metz, coll. 'Recherches linguistiques'nº 27 (I03-1 |9).

LeEMAN, D. (2008) « Prépositions du français : état des lieux », Introduction au présent numéro.

MeLIS, L. (2000) « La préposition en interposition », Actes du XXIle Congrès International de Linguistique et de Philologie Romanes, Tübingen, Niemeyer, VI (353-359).

MELIS, L. (2003) La préposition en français, Paris, Ophrys.

MulleR, C. (2004) « À propos de [pc z.] », in C. Leclère, E. Laporte, M. Piot \& M. Silberztein (éds) Lexique, Syntaxe et Lexique-grammaire. Syntax, Lexis \& Lexicon-Grammar. Papers in honour of Maurice Gross, Lingvisticae Investigationes Supplementa, 24, Amsterdam/Philadelphia, John Benjamins (439-453).

PICOCHE, J. (1993) Didactique du vocabulaire français, Paris, Nathan.

RIEGEL, M. et al. (1994) Grammaire méthodique du français, Paris, PUF.

SAUSSURE (de), F. (1972) Cours de linguistique générale, Paris, Payot (Ire éd. 1916).

TRÉVOUX (1955) Dictionnaire universel françois et latin vulgairement appelé Dictionnaire de Trévoux (Ire éd. 1732), Paris, Rollin.

VAGUeR, C. (2005) «Dans les + numéral: un déterminant de quantification faible? », Travaux de Linguistique, 50 ( I 13-129).

VAGUER, C. (2006) «Bibliographie générale: recueils et articles consacrés aux prépositions du français », Modèles linguistiques, 54, Tome XXVII-2 (I 7I-203).

VAGUeR, C. (2007) «Approches de la préposition hors 》, Communication à la Journée d'étude 'Les prépositions', Arras (30 mars), France [actes à par. dans Langages].

VAGUER, C. (2007) «Bibliographie: prépositions et locutions prépositionnelles », Modèles linguistiques, $55(|2|-|7|)$.

\section{Sources/Corpus}

[FRANTEXT] http://atilf.atilf.fr/frantext.htm

[GEOPO] Corpus libre de droits, accessible à l'adresse suivante: http://w3.univ-tlse2.fr/erss/textes/ pagespersos/hodac/Corpus/index.html

[UNITEX] http://www-igm.univ-mlv.fr/ unitex/

[TLFI] http://atilf.atilf.fr/tlf.htm 